\title{
Validación y adaptación española de la escala de resiliencia en el contexto deportivo (ERCD)
}

\section{Rubén TRIGUEROS*, Joaquín Francisco ÁLVAREZ*, José Manuel AGUILAR-PARRA*, Manuel ALCARÁZ* y Antonio ROSADO**.}

\author{
Universidad de Almería, España*. \\ Faculdade de Motricidade Humana, Universidade de Lisboa, Portugal**.
}

(Recibido, 7 Marzo 2017; Aceptad, 14 Mayo 2017)

\begin{abstract}
RESUMEN: Los deportistas a lo largo de su vida deportiva se pueden ver expuestos a situaciones que les son estresantes en mayor o menor medida, es precisamente esa capacidad para superarlos lo que se denomina resiliencia. En este estudio se pretende validar y adaptar la The Resilience Scale de Wagnild y Young (1993) a partir de la versión portuguesa desarrollada por Vigário et al., compuesto por 25 ítems. La muestra está conformada por 278 voleibolistas con una edad media de 20.9 años. El análisis factorial confirmatorio reveló unos índices de ajuste adecuados para el modelo de dos factores correlacionados y del modelo de orden superior de la ERCD, mostrándose la estructura invariante respecto al sexo. Los dos factores que integran la escala obtuvieron una alta consistencia interna. La ERCD se mostró como un instrumento psicométrico robusto y adecuado para medir en España la resiliencia en el contexto deportivo y de la actividad física.
\end{abstract}

Palabras Clave: Resiliencia, actividad física, deporte, análisis factorial.

Spanish validation and adaptation of the resilience scale in the sport context (ERCD)

\begin{abstract}
Athletes throughout their sports life can be exposed to situations that are stressful to a greater or lesser extent, it is precisely that ability to overcome what is called resilience. This study aims to validate and adapt Wagnild and Young's The Resilience Scale (1993) based on the Portuguese version developed by Vigário et al., composed of 25 items. The sample is made up of 278 volleyball players with a mean age of 20.9 years. Confirmatory factor analysis revealed adequate adjustment indices for the correlated two-factor model and the higher-order model of the ERCD, showing the invariant structure with respect to sex. The two factors that make up the scale obtained a high internal consistency. The ERCD was shown as a robust and adequate psychometric instrument to measure resilience in Spain in the context of sport and physical activity.
\end{abstract}

Key words: Resilience, physical activity, sport, factor analysis.

Correspondencia: Jose Manuel Aguilar-Parra. Universidad de Almería. Facultad de Educación. Departamento de Psicología. Carretera del Sacramento s/n. La Cañada de San Urbano (04120) Almería. (España). Mail: jmaguilar@ual.es. 
Desde una perspectiva eminentemente psicológica, la práctica deportiva se caracteriza por la continua exposición a una serie de circunstancias adversas y potencialmente estresantes a la cuales en mayor o menor medida, todo deportista se ve sometido (Sarkar \& Fletcher, 2014). De esta forma, incluso aquellos que desempeñan esta actividad de forma más exitosa, sufren en algún momento de su carrera algún episodio de consecuencias traumáticas, entre los cuales se encuentra la prácticamente inevitable experiencia de la derrota (Sagar, Lavallee, \& Spray, 2007; Wilson \& Kerr, 1999) o la lesión deportiva (Podlog, Dimmock, \& Miller, 2011; Salim, Wadey, \& Diss, 2015).

Precisamente a partir de la vivencia de este tipo de situaciones, así como de la consiguiente adaptación positiva que producida en respuesta a ésta, reconduce a la persona hacia su nivel normal de funcionamiento (Fletcher \& Sarkar, 2013; Gucciardi, Jackson, Coulter, \& Mallett, 2011; Luthar, Cicchetti, \& Becker, 2000), se articula la por otra parte controvertida conceptualización del término resiliencia, al cual la literatura científica ha llegado a caracterizar de forma indistinta como rasgo, proceso o resultado (Masten \& Tellegen, 2012; Pangallo, Zibarras, Lewis, \& Flaxman, 2014) o incluso, como la presencia simultánea de las tres anteriores condiciones (Masten, Best, \& Garmezy, 1990). A partir de dichos elementos, se puede deducir que el atleta resiliente, sería aquel que es capaz de mantener su nivel de funcionamiento habitual y un alto grado de eficacia en presencia de la adversidad (Gucciardi, Hanton, Gordon, Mallett, \& Temby, 2015; Sarkar \& Fletcher, 2014). De esta manera, la resiliencia se constituye como un elemento a partir del cual podría explicarse parte del rendimiento deportivo del atleta (Hosseini \& Besharat, 2010) y que podría contribuir por tanto al objetivo de desarrollar una carrera deportiva exitosa.

Sin embargo, pese a que este constructo ha sido ampliamente estudiado en entornos como el educativo (Miller, 2002), el militar (Hammermeister, Pickering, McGraw, \& Ohlson, 2012) o el laboral (Warner \& April, 2012), su estudio dentro del ámbito deportivo cuenta con poco más de una década de antigüedad (Galli \& Gonzalez, 2014; Secades et al., 2014). Durante este tiempo, las diversas investigaciones realizadas han puesto de relieve la importancia de la resiliencia en dicho contexto, habiendo sido relacionada con unos menores niveles de burnout (Gucciardi et al., 2011; Reche-García, Tutte-Vallarino, \& Ortín-Montero, 2014), unos estilos de afrontamiento positivo (Yi, Smith, \& Vitaliano, 2005) e incluso con unos mejores niveles de salud mental (Hosseini \& Besharat, 2010).

Gran parte de los estudios realizados en este ámbito, han planteado como principal objetivo la comprensión del comportamiento, pensamientos, creencias y emociones de aquellos atletas que previamente mostraron su capacidad para responder de forma eficiente en presencia de los diversos factores o elementos estresantes propios de la actividad deportiva (Galli \& Gonzalez, 2014), tarea para la cual se han empleado de forma predominante técnicas de tipo cualitativo como la entrevista (Brown, Lafferty, \& Triggs, 2015; Fletcher \& Sarkar, 2012; Galli \& Vealey, 2008; Machida, Irwin, \& Feltz, 2013; Sarkar, Fletcher, \& Brown, 2014). Recientemente y también mediante la aplicación de este tipo de técnicas, se ha estudiado este fenómeno desde una perspectiva colectiva en el ámbito de los deportes de equipo (Morgan, Fletcher, \& Sarkar, 2013, 2015).

A partir de estos estudios, se han establecido dos modelos teóricos explicativos de la resiliencia en el ámbito deportivo. Por un lado, el modelo de Galli \& Vealey, (2008), el cual, basado en el desarrollado previamente en el contexto educativo (Richardson, Neiger, Jensen, \& 
Kumpfer, 1990; Richardson, 2002) explica el fenómeno en función de un proceso metacognitivo desencadenado a partir de la exposición a la adversidad, en función de la cual se genera un estado de perturbación emocional, que finalmente es superado gracias a la existencia de una serie de condicionantes socioculturales (e.g. adecuado apoyo social) y personales (e.g. positividad, determinación, compromiso, competitividad, madurez, persistencia, pasión por la actividad), poniendo de relieve por tanto la importancia tanto de los factores ambientales como de los externos en el referido proceso y de otro, el modelo de Fletcher \& Sarkar $(2012,2014)$, centrado en las reacciones cognitivas de carácter adaptativo surgidas a partir de la interacción entre los factores estresores originados tanto en la faceta organizacional y competitiva de la actividad como en el propio ámbito personal y una serie de factores protectores de naturaleza psicológica entre los que destacan la personalidad positiva y el predominio de las formas más autodeterminadas de motivación, así como unos elevados niveles de confianza, concentración, y apoyo social percibido.

Por su parte, el estudio cualitativo de la resiliencia en este ámbito se ha visto condicionado por la imposibilidad de contar con una herramienta capaz de capturar la influencia de los factores contextuales específicos de este dominio, habiendo utilizado los investigadores interesados en emplear este tipo de técnicas en sus trabajos, herramientas cuya validez resultaría cuestionable atendiendo a dos aspectos fundamentales: a) haber sido desarrolladas en contextos muy diferentes al que nos ocupa (Gucciardi et al., 2011; Sarkar \& Fletcher, 2013), en los cuales los sujetos estudiados se vieron en la necesidad de exhibir un comportamiento catalogado como resiliente para volver a su anterior estado global de funcionamiento vital y no en respuesta a una situación concreta en el desempeños de una actividad voluntariamente escogida como podría ser la deportiva (Fletcher \& Sarkar, 2012); b) la disparidad de modelos teóricos en función de los cuales se construyeron las diversas herramientas, hecho que dificulta desde una perspectiva conceptual la elección inequívoca del instrumento psicométrico mediante el cual abordar la tarea en cuestión (Windle, Bennett, \& Noyes, 2011).

De esta forma, existen precedentes en el empleo de herramientas como la ConnorDavidson Resilience Scale (Connor \& Davidson, 2003) en muestras formadas por atletas en su versión original y completa de 25 items y 5 factores de orden inferior, los cuales son definidos como competencia personal, altos estándares y tenacidad (factor $\left.\mathrm{n}^{\circ} 1\right)$, confianza en el propio instinto, tolerancia de los afectos negativos y efecto fortalecedor del estrés (factor $\mathrm{n}^{\circ} 2$ ), aceptación positiva del cambio y seguridad en las relaciones (factor $\mathrm{n}^{\mathrm{0}} 3$ ), control (factor $\mathrm{n}^{\circ} 4$ ) e influencias espirituales (factor $\mathrm{n}^{\circ} 5$ ), como es el caso del trabajo de Hosseini \& Besharat (2010). Por su parte, (Gucciardi et al., 2011) llegaron a establecer incluso la superioridad en términos psicométricos de la versión unidimensional de 10 ítems previamente propuesta por CampbellSills, Cohan, \& Stein (2006) con respecto a la versión completa anteriormente citada en jugadores de cricket, empleando en dicho estudio dos muestras independientes de 321 y 199 adultos y adolescentes, respectivamente. Sin embargo y pese a que los resultados obtenidos en el trabajo de Gucciardi et al. viniesen a corroborar las adecuadas propiedades psicométricas de este instrumento anteriormente observadas en otros dominios (Windle et al., 2011), cabe destacar que dicha herramienta fue originalmente elaborada a partir de una concepción eminentemente de rasgo, más propia de la primera oleada de trabajos en este campo (Richardson, 2002; Wright, Masten, \& Narayan, 2013) muy alejada por tanto de la actual 
delimitación conceptual del constructo, tanto considerado de forma genérica (Luthar et al., 2000) como circunscrito al ámbito deportivo (Fletcher \& Sarkar, 2012; Galli \& Vealey, 2008).

Otro de los instrumentos empleados en este contexto, ha sido la Resilience Scale (RS; Wagnild \& Young, 1993), compuesta por 25 ítems redactados en sentido positivo a partir de la conceptualización del constructo obtenida tras la realización de un estudio cualitativo el cual empleó una muestra formada por mujeres mayores que habían superado con éxito algún tipo de evento traumático. Los autores matizaron los resultados a la luz de una revisión bibliográfica de de la literatura disponible, reseñando 5 principales atributos que emergieron como propios de la resiliencia: Perseverancia, Ecuanimidad, Significatividad, Auto-confianza y Sentirse bien solo, a partir de los cuales configuran la estructura final de la escala compuesta por dos factores: competencia personal y aceptación de uno mismo y de la vida. Este instrumento ha sido empleado en su versión portuguesa tanto en una muestra formada por 208 deportistas con discapacidad física (Cardoso \& Sacomori, 2014), como para comparar dos grupos de exdeportistas y no deportistas en el estudio de Cevada et al. (2012). Las propiedades de la versión española de esta escala, fueron originalmente estudiadas en el ámbito deportivo por Ruiz, De La Vega, Poveda, Rosado, \& Serpa (2012) empleando una muestra compuesta por 109 futbolistas jóvenes masculinos, obteniendo unas propiedades psicométricas adecuadas para la escala general y el primero de los factores, aunque no así para el segundo de los mismos, siendo sus índices de consistencia interna (Alpha de Cronbach) de .81, .76 y .49, respectivamente. Esta misma versión ha sido empleada con posterioridad por Reche García et al. (2014) en una muestra de 45 judokas, obteniendo valores de consistencia interna de $.89, .88$ y .59 para la escala general y cada uno de los dos factores de forma respectiva.

Pese a que trabajos como los de Gucciardi et al. (2011) y Ruiz et al. (2012) hayan contribuido a objetivo de contar con una herramienta destinada al estudio cuantitativo de la resiliencia en el deporte, ambos estudios no consideraron la posibilidad de haber adaptado el enunciado de sus ítems de forma que éstos reflejasen de manera específica y explícita, comportamientos producidos en respuesta ante situaciones de riesgo y potencialmente estresantes propias de este contexto ya que ambos estudios están focalizados hacia la vida diaria en general.

Establecida la importancia de profundizar en estudio de la resiliencia en el contexto deportivo, el presente trabajo pretende dar respuesta a la necesidad reiteradamente expuesta en la literatura de contar con herramientas de tipo cuantitativo que permitan profundizar en el estudio de este constructo (Fletcher \& Sarkar, 2012; Galli \& Vealey, 2008; Gucciardi et al., 2011; Sarkar \& Fletcher, 2013). En consecuencia, el principal objetivo del presente estudio es comprobar las propiedades psicométricas de una versión adaptada de forma específica al ámbito deportivo de la escala de Resiliencia de Wagnild \& Young (1993) a partir de la versión portuguesa desarrollada por Vigário et al. (2009). Adicionalmente pretendemos analizar las propiedades psicométricas de la estructura de orden superior denominado resiliencia con el fin de testar un constructo unitario que englobe los dos factores que integran la escala. A tal efecto, se pretende confirmar la estructura factorial y la consistencia interna de esta nueva versión del instrumento, comprobando igualmente la estabilidad temporal y la invarianza en función del sexo. Adicionalmente, mediante la inclusión de la variable de la ansiedad (Ramis, Torregrosa, Viladrich y Cruz, 2010) considerada de forma específica en el contexto deportivo, se busca obtener evidencias acerca de la validez convergente de la escala. En función de trabajos 
anteriores (Brown et al., 2015; Fletcher \& Sarkar, 2012; Sarkar \& Fletcher, 2014), se hipotetiza acerca de la existencia de una posible relación entre la resiliencia y la ansiedad, la cual podría resultar de carácter negativo.

\section{MÉTODO}

\section{Participantes}

En el presente estudio tomaron parte 278 voleibolistas semi-profesionales (174 varones y 104 mujeres), con edades comprendidas entre los 18 y 32 años $(M=21.04 ; D T=3.03)$, pertenecientes a diferentes clubes deportivos de la provincia de Almería.

\section{Medidas}

Escala de Resiliencia en el Contexto Deportivo (ERCD). Con el fin de medir la resiliencia en el contexto del deporte se validó y se adaptó The Resilience Scale de Wagnild y Young (1993) a partir de la versión portuguesa desarrollada por Vigário et al. (2009). Este cuestionario está encabezado por la sentencia "Considerando las vivencias deportivas responde tu grado de acuerdo o desacuerdo con las siguientes afirmaciones" y consta de 25 ítems repartidos en dos factores, diecisiete de ellos miden la competencia personal (eg." Soy disciplinado con el deporte que practico") y ocho de ellos la aceptación de uno mismo y de la vida (eg. "Siento que mi vida deportiva tiene sentido"). Los deportistas debían indicar su respuesta por medio de una escala Likert de 1 (en desacuerdo) a 7 (totalmente de acuerdo).

Cuestionario de Ansiedad Competitiva (SAS-2) de Ramis, et al. (2010) basado del cuestionario de Sport Anxiety Scale-2 de Smith, Smoll, Cumming \& Grossbard (2006). Este cuestionario está integrado por 15 ítems repartidos en 3 subescalas ansiedad somática, preocupación y desconcentración. Cada ítem se contesta a partir de una escala likert que va de 1 (nada) a 4 (mucho).

\section{Procedimiento}

Para poder llevar a cabo la validación y adaptación del cuestionario al contexto español se utilizó la estrategia de traducción inversa (Hambleton, 1996). Este proceso consiste en la traducción directa al español del cuestionario original, siendo realizado por un conjunto de expertos en traducción y que posteriormente otro grupo diferente de expertos tradujo a su idioma original. La bondad de la traducción se juzgó en función del grado de coincidencia con la versión original. La versión obtenida fue analizada por tres expertos (Lynn, 1986) en actividad física y deporte, de tal modo que se garantizara que los ítems obtenidos, estuvieran bien diseñados para medir el constructo que quería medirse, sin perder el significado original. Una vez obtenido el cuestionario, un grupo de expertos en la materia adaptaron los diferentes reactivos al deporte, puesto que la escala original se había diseñado para la vida.

Una vez obtenido el cuestionario se contactó con algunos responsables de clubes deportivos de voleibol habidos en la provincia de Almería, a lo que se les informó del objetivo de la investigación y solicitó su colaboración. Antes de administrar la escala a la totalidad de los participantes, ésta fue cumplimentada por un pequeño grupo de deportistas para asegurar la correcta comprensión de todos los ítems. La administración del cuestionario se realizó bajo la supervisión de un encuestador experto miembro del grupo de investigación, que explicó y 
solventó las dudas que surgieron al cumplimentarlo. El tiempo estimado para completar el cuestionario fue de 15 minutos.

\section{ANÁLISIS DE DATOS}

Con el fin de poder determinar la validez y fiabilidad de la Escala de Resiliencia al deporte en el contexto español se analizó las propiedades psicométricas del cuestionario. En primer lugar, con el fin de testar la estructura factorial del cuestionario además de la estructura de un modelo que englobara la resiliencia como factor de orden superior, se efectuó un análisis factorial confirmatorio (AFC). En segundo lugar, se efectuó un análisis multigrupo con el fin de analizar la invarianza respecto al género para poder determinar si el cuestionario es entendido por igual tanto por los chicos como por las chicas. Posteriormente, se efectuó un análisis estadístico-descriptivos y el análisis de consistencia interna a través de alfa de Cronbach. Para los análisis de datos se utilizaron los paquetes estadísticos SPSS 19.0 y AMOS 19.0 .

Dado que el coeficiente de Mardia resultó ser alto (124.844) para el AFC se utilizó el método de estimación de máxima verosimilitud junto con el procedimiento de bootstrapping. A pesar de la falta de normalidad los estimadores no resultaron afectados, por lo que se considerados robustos (Byrne, 2001). Con el objetivo de aceptar o rechazar el modelo testado, se tuvo en consideración un conjunto de índices de ajuste: $\chi 2 / g l$, CFI (Comparative Fit Index), TLI (Tucker Lewis Index), IFI (Incremental Fit Index), RMSEA (Root Mean Square Error of Approximation) más su intervalo de confianza (IC) al 90\%, y SRMR (Standardized Root Mean Square Residual). Dado que el $\chi^{2}$ es muy sensible al tamaño de la muestra (Jöreskog \& Sörbom, 1993), se empleó el $\chi 2 / g l$, considerándose aceptables valores inferiores a 5 (Bentler, 1989). Los índices incrementales (CFI, TLI e IFI) muestran un buen ajuste con valores iguales o superiores .90 (Schumacker \& Lomax, 1996), mientras que los índices de error (RMSEA y SRMR) se consideran aceptables con valores iguales o menores de .08 (Hu \& Bentler, 1999).

\section{RESULTADOS}

\section{Análisis factorial confirmatorio}

Los índices de ajuste del modelo testado (Figura 1) reveló unos índices de ajuste apropiados: $\chi^{2}(274 . N=278)=463.61, p<.001 ; \chi^{2} / g l=1.69 ; \mathrm{CFI}=.96$; TLI $=.96$; IFI $=.96$; RMSEA $=.07($ IC 90\% $=.060-.082)$; SRMR $=.029$. Los pesos de regresión estandarizados oscilaron entre .82 y .95 siendo estadísticamente significativos $(p<.001)$. En cuanto a las correlaciones entre los factores fue de .83 siendo estadísticamente significativo $(p<.001)$. 


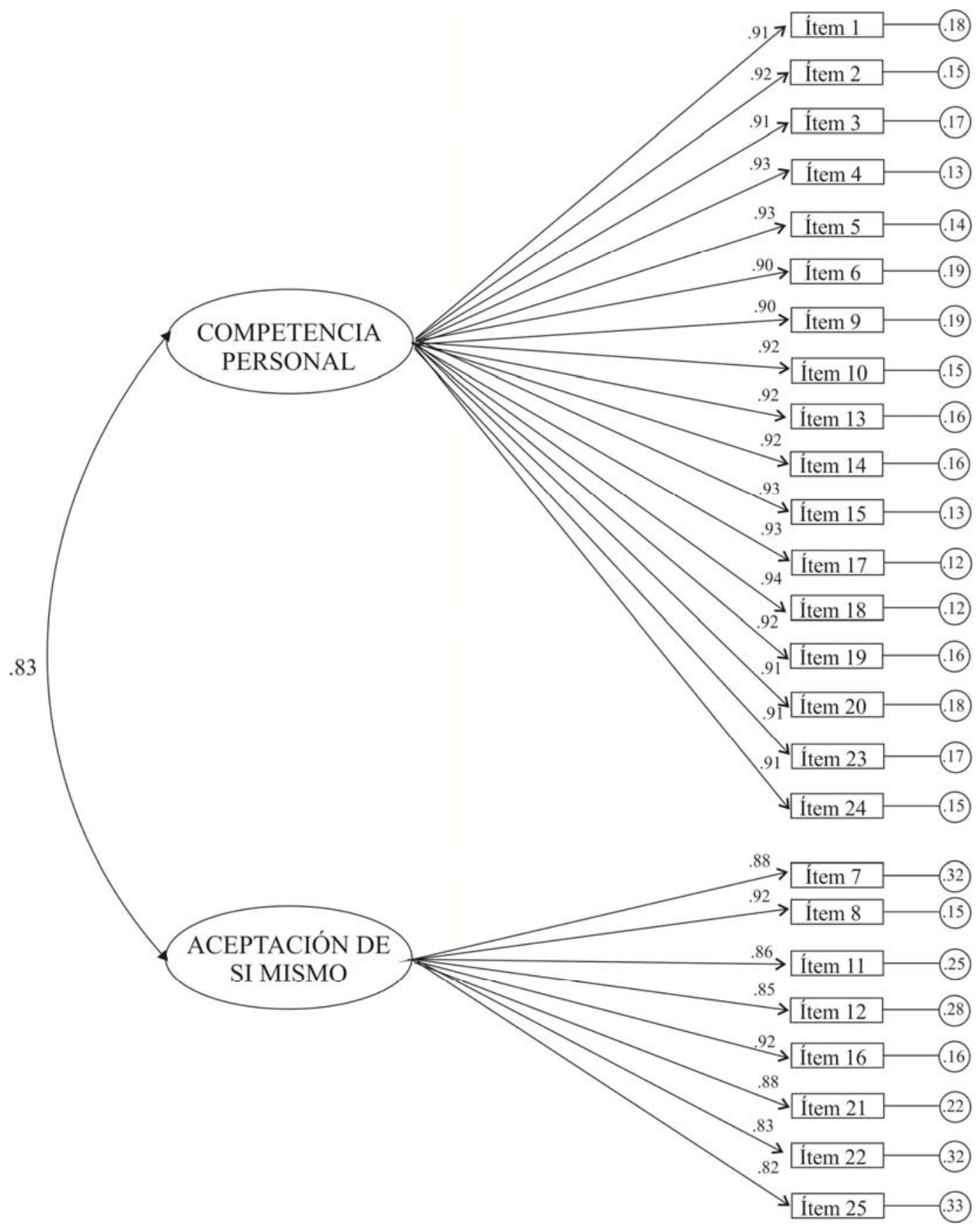

Figura 1. Análisis factorial confirmatorio de la ERCD. Las elipses representan los factores y los rectángulos representan los diferentes ítems. Las varianzas residuales se muestran en los círculos pequeños

Una vez determinado el modelo, se procedió a testar un modelo de orden superior (i.e., los dos factores de primer orden convergiendo en un factor de segundo orden superior denominado resiliencia). Los índices de ajuste de este modelo fueron ligeramente mejores: $\chi^{2}$ $(274 . N=278)=464.52, p<.001 ; \chi^{2} / g l=1.69 ; \mathrm{CFI}=.96 ; \mathrm{TLI}=.95 ; \mathrm{IFI}=.96 ; \mathrm{RMSEA}=.07$ (IC $90 \%=.060-.082) ;$ SRMR $=.028$ Todos los pesos de regresión estandarizados fueron significativos $(p<.001)$, siendo de .88 para competencia personal y de .95 aceptación de uno mismo. 


\section{Análisis de invarianza por sexo}

Para saber si la estructura factorial del modelo se muestra invariante respecto al género, se realizó un análisis multigrupo. Tal y como muestra la tabla 1 no se encontraron diferencias significativas en el estadístico $\chi 2$ entre el modelo no constreñido (Modelo 1) y los modelos con pesos de medida invariante (Modelo 2), pero si con el modelo de covarianzas estructurales invariantes (Modelo 3) y medidas residuales invariantes (Modelo 4). Dado es tos resultados apoyan la existencia de invariancia respecto al género en el modelo de cuatro factores de orden primario. En la tabla 1 también se muestran los diversos índices de ajuste para los seis modelos comparados dentro de la estructura de un factor de orden superior. No se encontraron diferencias significativas en el estadístico $\chi 2$ el modelo no constreñido no constreñido (Modelo 1) y los modelos con pesos de medida invariante (Modelo 2), pero si con el modelo de covarianzas estructurales invariantes (Modelo 3) y medidas residuales invariantes (Modelo 4), residuales estructurales invariantes (Modelo 5), y medidas residuales invariantes (Modelo 6). La ausencia de diferencias significativas entre el modelo 1 y el modelo 2 supone un criterio mínimo aceptable para manifestar que la estructura del modelo es invariante respecto al género (Marsh, 1993).

Tabla 1: Análisis de Invarianza por Sexo

\begin{tabular}{ccccccccccc}
\hline Modelos & $\chi^{2}$ & $g l$ & $\chi^{2} / g l$ & $\Delta \chi^{2}$ & $\Delta g l$ & CFI & TLI & IFI & RMSEA (IC 90\%) & SRMR \\
\hline Modelo 1 & 918,62 & 548 & 1,68 & - & - &, 93 &, 92 &, 93 &, $071(, 062-, 078)$ &, 055 \\
Modelo 2 & 945,88 & 571 & 1,66 & 27,27 & 23 &, 93 &, 92 &, 93 &, $069(, 062-, 077)$ &, 054 \\
Modelo 3 & 992,23 & 574 & 1,73 & $73,61 * *$ & 26 &, 92 &, 91 &, 92 &, $073(, 065-, 081)$ &, 068 \\
Modelo 4 & 1009,75 & 599 & 1,69 & $91,13^{* *}$ & 51 &, 92 &, 92 &, 92 &, $071(, 063-, 079)$ &, 068 \\
\hline
\end{tabular}

Modelo de un factor de orden superior

\begin{tabular}{ccccccccccc}
\hline Modelos & $\chi^{2}$ & $g l$ & $\chi^{2} / g l$ & $\Delta \chi^{2}$ & $\Delta g l$ & CFI & TLI & IFI & RMSEA (IC 90\%) & SRMR \\
\hline Modelo 1 & 918,62 & 548 & 1,68 & - & - &, 93 &, 92 &, 93 &, $071(, 062-, 078)$ &, 056 \\
Modelo 2 & 945,88 & 571 & 1,66 & 27,27 & 23 &, 93 &, 92 &, 93 &, $069(, 062-, 077)$ &, 057 \\
Modelo 3 & 977,76 & 572 & 1,71 & $59,14^{* *}$ & 24 &, 92 &, 91 &, 92 &, $072(, 064-, 080)$ &, 068 \\
Modelo 4 & 992,23 & 574 & 1,73 & $73,61 * *$ & 26 &, 92 &, 91 &, 92 &, $073(, 065-, 081)$ &, 068 \\
Modelo 5 & 1000,75 & 585 & 1,71 & $81,13^{* *}$ & 41 &, 92 &, 92 &, 92 &, $071(, 063-, 079)$ &, 072 \\
Modelo 6 & 1009,75 & 599 & 1,69 & $91,13^{* *}$ & 51 &, 92 &, 92 &, 92 &, $071(, 063-, 079)$ &, 078 \\
\hline
\end{tabular}

$* * p<, 001$. Nota. Modelo $1=$ modelo sin restricciones; Modelo $2=$ modelo con pesos de medida invariantes; Modelo 3 = modelo con covarianzas estructurales invariantes; Modelo $4=$ modelo con residuos de medida invariantes; Modelo $5=$ residuales estructurales invariantes, y Modelo $6=$ medidas residuales invariantes.

\section{Análisis de fiabilidad}

Con el fin de obtener evidencias de fiabilidad de la escala, se realizó un análisis de consistencia interna y un análisis de estabilidad temporal de los diferentes factores que 
componen la ERCD. El análisis de consistencia interna reflejó unos valores de alfa de Cronbach de .96 para aceptación de sí mismo y de la vida y de .98 para competencia personal.

Para analizar la estabilidad temporal de la ERCD usamos una muestra independiente, a la que administramos la escala en dos ocasiones con un intervalo de dos semanas entre la primera y segunda toma de datos. El coeficiente de correlación intraclase (CCI) y los intervalos de confianza (IC) fueron calculados con objeto de evaluar la estabilidad temporal de cada una de las subescalas. Las medias de la aceptación de sí mismo y de la vida fueron de 5.66 $(\mathrm{DT}=.94)$ y $5.77(\mathrm{DT}=.72)$ con un CCI de $91(\mathrm{IC}=.92-.95)$ y para competencia personal fue de $5.13(\mathrm{DT}=1.17)$ y $5.65(\mathrm{DT}=1.18)$ con un CCI de $.92(\mathrm{IC}=.96-.80)$.

En cuanto al modelo de orden superior, el análisis de consistencia interna reflejó unos valores de alfa de Cronbach de .96 y el análisis de estabilidad temporal con un CCI .90 $(\mathrm{IC}=.89-.92)$.

\section{Estadísticos Descriptivos y Correlaciones Bivariadas Entre las Variables}

Tal y como muestra la Tabla 2 los participantes en el estudio obtuvieron una mayor puntuación media para competencia personal 5.64. Las correlaciones bivariadas entre ambos factores que integran la ERDC muestran como son positivas entre sí .82 lo que indica que si uno de los factores aumenta el otro también lo hará.

Tabla 2. Estadísticos Descriptivos y Correlaciones Bivariadas entre variables

\begin{tabular}{lcccccc}
\hline Factores & $M$ & $D T$ & Rango & 1 & 2 & 3 \\
\hline 1. Factor uno resiliencia & 5.64 & 1.14 & $1-7$ & $.82 * * *$ & $-.21^{* * *}$ \\
2. Factor dos resiliencia & 5.58 & 1.07 & $1-7$ & & $-.14^{* * *}$ \\
3. Ansiedad & 1.85 & .26 & $1-4$ & & \\
\hline$* * * p<, 001$. Nota: Factor uno resiliencia = competencia personal; Factor dos \\
resiliencia $=$ aceptación de si mismo y de la vida. \\
\hline
\end{tabular}

\section{Análisis de validez de criterio}

Con el fin de analizar la validez de criterio de la ERDC se realizó un análisis de regresión lineal por el cual se introdujo como variable dependiente la ansiedad y los factores que integran la resiliencia como variable independiente.

Los resultados (Tabla 3) revelaron que la competencia personal predice de forma significativa la ansiedad, con un peso de regresión positivo y proporcionando una varianza explicada del $11 \%$.

Tabla 3. Análisis de Regresión Lineal de los factores que integran la resiliencia que predicen la ansiedad

\begin{tabular}{lcccc}
\hline Variables & $F$ & $R^{2}$ & $\beta$ & $t$ \\
\hline & 8,99 &, $11^{* * *}$ & & \\
Competencia Personal & & &,- 01 &,- 11 \\
Aceptación de sí mismo & &,- 11 & $-4,55^{* * *}$ \\
\hline$* * * p<, 001$. & & & \\
\hline
\end{tabular}




\section{DISCUSIÓN}

El objetivo del presente estudio consiste en la validación y adaptación The Resilience Scale de Wagnild \& Young (1993) a partir de la versión portuguesa desarrollada por Vigário et al. (2009), siguiendo un proceso de traducción y adaptación y posteriormente analizando sus propiedades psicométricas en un estudio con deportistas españoles, en este caso, voleibolistas semiprofesionales. Los resultados del estudio han mostrado que la ERCD es un instrumento que muestra evidencias de validez y fiabilidad para medir los diferentes aspectos conductuales de la resiliencia en España especifico para el contexto deportivo. Este instrumento podría ayudar a entender mejor la superación de las consecuencias negativas que están presentes a lo largo de la vida deportiva de los deportistas.

Los resultados de este estudio revelaron que la estructura factorial de la ERCD apoya el modelo de dos factores. Este resultado está en consonancia con estudios anteriores en contextos diferentes. Los resultados del análisis de las correlaciones bivariadas no mostraron correlaciones muy altas entre ambos factores, apoyando la validez discriminante entre las diferentes subescalas. En lo referido al modelo de orden superior, con el nombre resiliencia, fue también apoyada a través del AFC. Este modelo resulta de especial interés ya que apoya el uso de un valor global de la resiliencia en futuras investigaciones, englobando los factores que integran la escala. Este uso puede darse en investigación que requieran de modelos complejos donde se establezcan relaciones entre varios constructos. Igualmente, teóricamente viene estando justificado ya que ambos factores que integran la escala de resiliencia tiende a actuar de forma muy estrecha, dependiendo uno del otro (Wagnild \& Young, 1993).

Los resultados de consistencia interna arrojaron valores alfa de Cronbach superiores a .85 en cada una de las dos subescalas, así como el modelo de orden superior, arrojando un valor superior a .95. Estos datos está en consonancia con las investigaciones de Wagnild \& Young (1993) y de Vigário et al. (2009) donde se obtuvieron unos valores de alfa de Cronbach superior a .70. Además, la escala de Resiliencia en el Deporte ha mostrado una adecuada estabilidad temporal en cada una de las dos subescalas.

Los análisis multigrupo realizados mostraron que tanto la estructura de un modelo de dos factores como un modelo de orden superior de la Escala de Resiliencia fueron invariantes respecto al género. Este resultado posibilita que futuros estudios puedan establecer la comparación de medias entre los hombres y mujeres. Sin embargo, futuras investigaciones deberían de examinar la invarianza de la ERCD respecto a otras variables. Por ejemplo, la invarianza por edad o respecto a los diferentes deportes que en este estudio no ha sido analizada debido a que la muestra de deportistas representó poca variabilidad.

Entendemos por lo tanto que estos resultados proporcionan evidencia en favor de la solidez de la estructura de esta medida y revelan que la versión adaptada consigue replicar fielmente la estructura teórica original. A partir de ahora se podrá contar con una herramienta corta, de fácil aplicación para los administradores y de fácil comprensión para los deportistas que pudiera evaluar la resiliencia específica en el deporte. La versión adaptada de resiliencia en el deporte ha revelado datos satisfactorios ajustándose al modelo teórico subyacente $\mathrm{y}$ mostrando alta consistencia interna y validez, y dado que la resiliencia se asocia de forma positiva con el rendimiento deportivo y el bienestar psicológico y, una relación negativa con los 
trastornos psicológicos (Hosseini \& Besharat, 2010) resultará interesante la futura utilización de la ERCD en el ámbito del deporte en todos sus niveles.

\section{ESCALA DE RESILIENCIA EN EL CONTEXTO DEPORTIVO}

1. Cuando me planteo objetivos, sigo adelante hasta conseguirlos

2. Siento que puedo resolver problemas que se me plantean durante el entrenamiento

3. Creo en mí más que en nadie

4. Para mí es importante mantener el interés en el entrenamiento

5. Puedo afrontar los problemas del entrenamiento yo solo, si es necesario

6. Me siento orgulloso de haber superado las dificultades del entrenamiento

7. Suelo afrontar problemas con calma

8. Soy amigo de mi mismo

9. Siento que puedo manejar varios problemas a la vez

10. Soy decidido

11. Rara vez pienso que no lograre los objetivos que me he planteado

12. Realizo las cosas en su debido tiempo

13. Puedo lidiar con situaciones difíciles

14. Soy disciplinado

15. Tiendo a mantener el interés por el entrenamiento

16. Por lo general, encuentro muchas razones para divertirme

17. Creer en mí mismo me ayuda a superar los momentos difíciles

18. En una situación difícil, soy alguien en quien pueden confiar el resto de la gente

19. Por lo general, puedo resolver una misma situación de formas diferentes

20. A veces me obligo a hacer cosas que no se si quiero hacer

21. Siento que mi entrenamiento tiene sentido

22. No suelo insistir en cosas que no puedo cambiar

23. Cuando me encuentro en una situación difícil, por lo general suelo encontrar una salida

24. Tengo energía suficiente para hacer lo que tengo que hacer

25. No tengo ningún problema si hubiera gente que no es de mi agrado

Los deportistas debían indicar su respuesta por medio de una escala Likert de 1 (en desacuerdo) a 7 (totalmente de acuerdo).

\section{REFERENCIAS}

Bentler, P. M. (1989). EQS structural equations program manual. Los Angeles: BMDP Statistical Software.

Brown, H. E., Lafferty, M. E., \& Triggs, C. (2015). In the face of adversity: Resiliency in winter sport athletes. Science \& Sports, 1-13. doi:10.1016/j.scispo.2014.09.006

Campbell-Sills, L., Cohan, S. L., \& Stein, M. B. (2006). Relationship of resilience to personality, coping, and psychiatric symptoms in young adults. Behaviour Research and Therapy, 44(4), 585-599.

Cardoso, F. L., \& Sacomori, C. (2014). Resilience of athletes with physical disabilities: A cross-sectional study. Revista de Psicologia Del Deporte, 23(1), 15-22. 
Cevada, T., Cerqueira, L. S., Sales de Moraes, H., Meireles Dos Santos, T., Monteiro Saboia, F. A., \& Camaz Deslandes, A. (2012). Relationship between sport, resilience, quality of life, and anxiety. Revista de Psiquiatria ..., 39(3), 85-89. Retrieved from http://www.scielo.br/scielo.php?pid=S0101$60832012000300003 \&$ script $=$ sci_arttext\&tlng=pt

Connor, K. M., \& Davidson, J. R. T. (2003). Development of a new resilience scale: The Connor-Davidson resilience scale (CD-RISC). Depression and Anxiety, 18(2), 76-82. doi:10.1002/da.10113

Deci, E. L., \& Ryan, R. M. (2000). The "What" and "Why" of Goal Pursuits: Human Needs and the Self-Determination of Behavior. Psychological Inquiry, 11(4), 227-268. doi:10.1207/S15327965PLI1104_01

Fletcher, D., \& Sarkar, M. (2012). A grounded theory of psychological resilience in Olympic champions. Psychology of Sport and Exercise, 13(5), 669-678. doi:10.1016/j.psychsport.2012.04.007

Fletcher, D., \& Sarkar, M. (2013). Psychological Resilience. European Psychologist, 18(1), 12-23. doi:10.1027/1016-9040/a000124

Galli, N., \& Gonzalez, S. P. (2014). Psychological resilience in sport: A review of the literature and implications for research and practice. International Journal of Sport and Exercise Psychology, (October 2014), 1-15. doi:10.1080/1612197X.2014.946947

Galli, N., \& Vealey, R. S. (2008). “ Bouncing Back " From Adversity: Athletes ' Experiences of Resilience. The Sport Psychologist, 22(2002), 316-335.

Gucciardi, D. F., Hanton, S., Gordon, S., Mallett, C. J., \& Temby, P. (2015). The concept of mental toughness: tests of dimensionality, nomological network, and traitness. Journal of Personality, 83(1), 26-44. doi:10.1111/jopy.12079

Gucciardi, D. F., Jackson, B., Coulter, T. J., \& Mallett, C. J. (2011). The Connor-Davidson Resilience Scale (CD-RISC): Dimensionality and age-related measurement invariance with Australian cricketers. Psychology of Sport and Exercise, 12(4), 423-433. doi:10.1016/j.psychsport.2011.02.005

Hammermeister, J., Pickering, M. A., McGraw, L., \& Ohlson, C. (2012). The Relationship Between Sport Related Psychological Skills and ... Journal of Sport Behavior, 35(1), $40-60$.

Hosseini, S. A., \& Besharat, M. A. (2010). Relation of resilience whit sport achievement and mental health in a sample of athletes. Procedia - Social and Behavioral Sciences, 5, 633-638. doi:10.1016/j.sbspro.2010.07.156

Hu, L., \& Bentler, P. M. (1999). Cutoff criteria for fit indexes in covariance structure analysis: Conventional criteria versus new alternatives. Structural Equation Modeling, 6, 1-55. http://dx.doi.org/10.1080/10705519909540118

Jöreskog, K. G., \& Sörbom, D. (1993). LISREL 8: Structural equation modeling with the SIMPLIS command language. Chicago: Scientific Software.

Luthar, S. S., Cicchetti, D., \& Becker, B. (2000). The Construct of Resilience: A Critical Evaluation and Guidelines for Future Work. Child Development, 71(3), 543-562.

Machida, M., Irwin, B., \& Feltz, D. (2013). Resilience in Competitive Athletes With Spinal Cord Injury The Role of Sport Participation. Qualitative Health Research, 23(8), 10541065. 
Marsh, H. W. (1993). The multidimensional structure of academic self-concept: Invariance over gender and age. American Educational Research Journal, 30, 841-860. http://dx.doi.org/10.3102/00028312030004841

Masten, A. S., Best, K. M., \& Garmezy, N. (1990). Resilience and development: Contributions from the study of children who overcome adversity. Development and Psychopathology, 2(04), 425-444.

Masten, A. S., \& Tellegen, A. (2012). Resilience in developmental psychopathology: contributions of the Project Competence Longitudinal Study. Development and Psychopathology, 24(2), 345-61. doi:10.1017/S095457941200003X

Miller, M. (2002). Resilience elements in students with learning disabilities. Journal of Clinical Psychology, 58(3), 291-298.

Morgan, P. B. C., Fletcher, D., \& Sarkar, M. (2013). Defining and characterizing team resilience in elite sport. Psychology of Sport and Exercise, 14(4), 549-559. doi:10.1016/j.psychsport.2013.01.004

Morgan, P. B. C., Fletcher, D., \& Sarkar, M. (2015). Understanding team resilience in the world's best athletes: A case study of a rugby union World Cup winning team. Psychology of Sport and Exercise, 16, 91-100. doi:10.1016/j.psychsport.2014.08.007

Pangallo, A., Zibarras, L., Lewis, R., \& Flaxman, P. (2014). Psychological Assessment Resilience Through the Lens of Interactionism: A Systematic Review Resilience Through the Lens of Interactionism: A Systematic Review.

Podlog, L., Dimmock, J., \& Miller, J. (2011). A review of return to sport concerns following injury rehabilitation: Practitioner strategies for enhancing recovery outcomes. Physical Therapy in Sport, 12(1), 36-42. doi:10.1016/j.ptsp.2010.07.005

Ramis, Y., Torregrosa, M., Viladrich, C., \& Cruz, J. (2010). Adaptación y validación de la versión española de la Escala de Ansiedad Competitiva SAS-2 para deportistas de iniciación. Psicothema, 22(4), 1004-1009.

Reche García, C., Tutte Vallarino, V., \& Ortín Montero, F. J. (2014). Resiliencia, Optimismo y Burnout en Judokas de competición uruguayos. Revista Iberoamericana de Psicología Del Ejercicio Y El Deporte, 9(2), 271-286.

Richardson, G. E. (2002). The metatheory of resilience and resiliency. Journal of Clinical Psychology, 58(3), 307-321. doi:10.1002/jclp.10020

Richardson, G. E., Neiger, B. L., Jensen, S., \& Kumpfer, K. L. (1990). The resiliency model. Health Education, 21(6), 33-39.

Ruiz, R., De La Vega, R., Poveda, J., Rosado, A., \& Serpa, S. (2012). Análisis psicométrico de la escala de resiliencia en el deporte del fútbol. Revista de Psicologia Del Deporte, 21(1), 143-151.

Sagar, S. S., Lavallee, D., \& Spray, C. M. (2007). Why young elite athletes fear failure: Consequences of failure. Journal of Sports Sciences, 25(11), 1171-1184.

Salim, J., Wadey, R., \& Diss, C. (2015). Examining the Relationship between Hardiness and Perceived Stress-Related Growth in a Sport Injury Context. Psychology of Sport and Exercise, 19, 10-17. doi:10.1016/j.psychsport.2014.12.004

Sarkar, M., \& Fletcher, D. (2013). How Should We Measure Psychological Resilience in Sport Performers? Measurement in Physical Education and Exercise Science, 17(4), 264-280. doi:10.1080/1091367X.2013.805141 
Sarkar, M., \& Fletcher, D. (2014). Psychological resilience in sport performers: a review of stressors and protective factors. Journal of Sports Sciences, 32(15), 37-41. doi:10.1080/02640414.2014.901551

Sarkar, M., Fletcher, D., \& Brown, D. J. (2014). What doesn't kill me. .: Adversity-related experiences are vital in the development of superior Olympic performance. Journal of Science and Medicine in Sport, 18(4), 475-479. doi:10.1016/j.jsams.2014.06.010

Schumacker, R. y Lomax, R.G. (1996) A Beginner's Guide to Structural Equation Modelling. Lawrence Erlbaum Associates Inc., Mahwah-NJ.

Secades, X. G., Molinero, O., Barquín, R. R., Salguero, A., Vega, R. D. La, \& Márquez, S. (2014). La resiliencia en el deporte: fundamentos teóricos, instrumentos de evaluación y revisión de la literatura. Cuadernos de Psicología Del Deporte, 14(3), 83-92. Retrieved from http://revistas.um.es/cpd/article/view/211291

Smith, R.E., Smoll, F.L., Cumming, S.P., y Grossbard, J.R. (2006). Measurement of multidimensional sport performance anxiety in children and adults: The sport anxiety scale-2. Journal of Sport and Exercise Psychology, 28, 479-501.

Wagnild, G., \& Young, H. M. (1993). Development and psychometric evaluation of the Resilience Scale. Journal of Nursing Measurement, 1(2), 165-178.

Warner, R., \& April, K. (2012). Building personal resilience at work. Effective Executive, $X V(4), 53-68$. doi:10.1179/1743275813Y.0000000026

Wilson, G. V., \& Kerr, J. H. (1999). Affective responses to success and failure: : A study of winning and losing in competitive rugby. Personality and Individual Differences, 27(1), 85-99. doi:10.1016/S0191-8869(98)00226-8

Windle, G., Bennett, K. M., \& Noyes, J. (2011). A methodological review of resilience measurement scales. Health and Quality of Life Outcomes, 9(1), 8. doi:10.1186/14777525-9-8

Wright, M. O. D., Masten, A. S., \& Narayan, A. J. (2013). Resilience process in development. In S. Goldstein \& R. B. Brooks (Eds.), Handbook of Resilience in Children (pp. 15-37). New York: Springer US. doi:10.1007/978-1-4614-3661-4

Yi, J. P., Smith, R. E., \& Vitaliano, P. P. (2005). Stress-resilience, illness, and coping: a person-focused investigation of young women athletes. Journal of Behavioral Medicine, 28(3), 257-265. 